\title{
VARIABLE GRAVITY EFFECTS ON THERMAL INSTABILITY OF NANOFLUID IN ANISOTROPIC POROUS MEDIUM
}

\author{
R. CHAND* \\ Department of Mathematics, Government P.G. College \\ Dhaliara, Himachal Pradesh, INDIA \\ E-mail: rameshnahan@yahoo.com \\ G.C. RANA \\ Department of Mathematics, NSCBM Govt. P.G. College \\ Hamirpur, Himachal Pradesh, INDIA \\ S. KUMAR \\ Department of Mathematics, Govt. P.G. College \\ Mandi, Himachal Pradesh, INDIA
}

\begin{abstract}
In this paper, we study the effects of variable gravity on thermal instability in a horizontal layer of a nanofluid in an anisotropic porous medium. Darcy model been used for the porous medium. Also, it incorporates the effect of Brownian motion along with thermophoresis. The normal mode technique is used to find the confinement between two free boundaries. The expression of the Rayleigh number has been derived, and the effects of variable gravity and anisotropic parameters on the Rayleigh number have been presented graphically.
\end{abstract}

Key words: nanofluid, anisotropic porous medium, Rayleigh number, variable gravity.

\section{Introduction}

Theoretical and experimental results on the stability of cellular convection of a fluid layer in a nonporous medium, in the presence of rotation and magnetic field, have been given by Chandrasekhar (1981). Lapwood (1948) studied the convective flow in a porous medium using the linearized stability theory. The Rayleigh instability of a thermal boundary layer in the flow through a porous medium was considered by Wooding (1960). The problem of convective instability of a horizontal fluid saturated porous layer was extensively investigated and the growing volume of work devoted to this area is well documented by Ingham and Pop (1998), Nield and Bejan (2006), and Vafai (2000).

Nanofluids have novel properties that make them potentially useful in many applications in heat transfer, including microelectronics, fuel cells, pharmaceutical processes and hybrid powered engines, domestic refrigerators, chillers, heat exchangers and nuclear reactors, in grinding, in machining, in space, defense and ships, and in boiler flue gas temperature reduction. They exhibit enhanced thermal conductivity and the convective heat transfer coefficient compared to the base fluid. A nanofluid is a mixture of the base fluid such as water or ethylene glycol and other coolants, oil and other lubricants, bio-fluids, polymer solutions etc. with a very small amount of nanoparticles or nanofibres such as metals or metallic oxides $\left(\mathrm{Cu}, \mathrm{Cuo}, \mathrm{Al}_{2} \mathrm{O}_{3}\right)$, metal carbides $(\mathrm{SiC})$, nitrides (AlN, SiN) or metals ( $\left.\mathrm{Al}, \mathrm{Cu}\right)$, etc., having dimensions from 1 to $100 \mathrm{~nm}$. It was Choi (1995) who first proposed the term "nanofluid."

Convection of nanofluids based on Buongiorno's model has attracted great interest. Buongiorno (2006) noted that the nanoparticles absolute velocity can be viewed as the sum of the base fluid velocity and

\footnotetext{
* To whom correspondence should be addressed
} 
a relative (slip) velocity. Vadasz (2006) studied heat conduction in nanofluid suspensions whereas Alloui et al. (2010) studied the natural convection of nanofluids in a shallow cavity heated from below. The Bénard problem (the onset of convection in a horizontal layer uniformly heated from below) for a nanofluid was studied by Tzou (2008a; b). Dhananjay et al. (2010) studied the Rayleigh - Bénard convection in a nanofluid by the weighted residual method. Nield and Kuznetsov (2010; 2011a), Chand (2012) studied convection in nanofluid. The thermal instability of a nanofluid in a porous medium was studied by Nield and Kuznetsov (2009), Nield and Kuznetsov (2011b), Kuznetsov and Nield (2011a; b; c), and Chand and Rana (2012).

All the above-mentioned studies have been concerned with homogeneous isotropic porous structures. The geological and pedological processes rarely form isotropic media as is usually assumed in transport studies. In geothermal systems with a ground structure composed of many strata of different permeabilities, the overall horizontal permeability may be up to ten times as large as the vertical component. Processes such as sedimentation, compaction, frost action, and reorientation of the solid matrix are responsible for the creation of anisotropic natural porous media. Anisotropy can also be a characteristic of an artificial porous material like pelleting used in chemical engineering processes and fiber materials used in insulating purposes. The studies of anisotropy and heterogeneous character of porous media can be found in the book by Nield and Bejan (2006). Recently, many authors have studied the effect of anisotropy on the onset of convection in a porous layer Malashetty et al. (2009).

The idealization of uniform gravity assumed in theoretical investigations, although valid for laboratory purposes can scarcely be justified for large-scale convection phenomena occurring in the atmosphere, the ocean or mantle of the Earth. It then becomes imperative to consider gravity as a variable quantity varying with distance from the surface or reference point. Pradhan et al. (1989) studied the thermal instability of a fluid layer in a variable gravitational field while Alex et al. (2001) studied the variable gravity effects on the thermal instability in a porous medium with internal heat source and inclined temperature gradient. Recently, Chand $(2011 ; 2012)$ studied the variable gravity effects on the thermal instability in the fluid layer.

In the present paper, an attempt has been made to study variable gravity effects on the thermal instability of a nanofluid in an anisotropic porous medium.

\section{Mathematical formulations of the problem}

Consider a horizontal anisotropic porous layer of a nanofluid of thickness ' $d$ ' bounded by plane $z=0$ and $z=d$, heated from below. Each boundary surface is assumed to be impermeable and perfectly thermal conducting. The porous medium is assumed to be possessing isotropy in the horizontal plane in mechanical property. The temperature $T$ and volumetric fraction $\varphi$ of nano particles are constant at the boundaries and taken to be $T_{0}$ and $\varphi=\varphi_{0}$ at $z=0$ and $T_{1}$ and $\varphi_{1}$ at $z=d,\left(T_{0}>T_{1}\right)$. The reference temperature is taken to be $T_{1 .}$

The fluid layer is acted upon by the gravity force $g$ and it is assumed that the gravity force vector varies linearly with $z$, i.e., $\boldsymbol{g}=[1+\delta h(z)] g \hat{\boldsymbol{k}}$, where $\delta h(z)$ is the variable gravity parameter. For simplicity, Darcy's law is assumed to hold and the Oberbeck-Boussinesq condition is employed. Nanoparticles are suspended in the nanofluid using either surfactant or surface charge technology, preventing the agglomeration and deposition of these on the porous matrix.

Thus the equations of continuity and motion for the nanofluid in an anisotropic porous medium (Chandersekhar 1961; Nield and Kuznetsov, 2009; Nield and Bejan, 2006; Malashetty and Kollur, 2011) are

$$
\begin{aligned}
& \nabla \cdot \boldsymbol{q}=0, \\
& 0=-\nabla p+\varphi \rho_{p} \hat{\boldsymbol{k}}+(1-\varphi)\left\{\rho\left(1-\alpha\left(T-T_{0}\right)\right)\right\} \boldsymbol{g}-\mu \boldsymbol{K} \cdot \boldsymbol{q}
\end{aligned}
$$


where $q(u, v, w)$ is the Darcy velocity vector, $\rho$ the density of the nanofluid, $\varepsilon$ the porosity, $p$ the hydrostatic pressure, $\varphi$ the volume fraction of the nanoparticles, $\rho_{p}$ is the density of nano particles, $\alpha$ is the coefficient of thermal expansion, $\mu$ is viscosity and $\boldsymbol{K}\left(=K_{x}^{-1}(\hat{i} \hat{i}+\hat{j} \hat{j})+K_{z}^{-1}(\hat{k} \hat{k})\right)$ the anisotropic permeability tensor, $K_{x}$, denotes the permeability in the $x$ - direction and $K_{z}$ permeability in the $z$-direction.

The energy equation for the nanofluid in isotropic porous medium is

$$
\sigma \frac{\partial T}{\partial t}+\boldsymbol{q} \cdot \nabla T=\kappa_{T} \nabla^{2} T+\left(D_{B} \nabla \varphi \cdot \nabla T+\frac{D_{T}}{T_{1}} \nabla T \cdot \nabla T\right)
$$

where $\kappa_{T}$ is the effective thermal diffusivity tensor.

The equation of continuity for the nanoparticles is

$$
\frac{\partial \varphi}{\partial t}+\frac{1}{\varepsilon} \boldsymbol{q} \cdot \nabla \varphi=D_{B} \nabla^{2} \varphi+\frac{D_{T}}{T_{b}} \nabla^{2} T
$$

where $D_{B}$ is the Brownian diffusion coefficient, given by Einstein-Stokes equation and $D_{T}$ is the thermoporetic diffusion coefficient of the nanoparticles.

Since the fluid under consideration is confined between two horizontal planes $z=0$ and $z=d$, on these two planes certain boundary conditions must be satisfied. We take the case of free-free surface and assume the temperature and volumetric fraction of the nano particles are constant and the boundary conditions (Chandrasekhar, 1961; Nield and Bejan, 2006) are

$$
w=0, \quad T=T_{0} \quad \varphi=\varphi_{0} \quad \text { at } \quad z=0,
$$

and $\quad w=0, \quad T=T_{1} \quad \varphi=\varphi_{1} \quad$ at $\quad z=d$.

We introduce non-dimensional variables as

$$
\begin{array}{ll}
\left(x^{\prime}, y^{\prime}, z^{\prime},\right)=\left(\frac{x, y, z}{d}\right), \quad\left(u^{\prime}, v^{\prime}, w^{\prime},\right)=\left(\frac{u, v, w}{\kappa_{T z}}\right) d, \quad t^{\prime}=\frac{t \kappa_{T z}}{\sigma d^{2}}, \quad p^{\prime}=\frac{p k}{\mu \kappa_{T z}} \\
\varphi^{\prime}=\frac{\left(\varphi-\varphi_{0}\right)}{\left(\varphi_{1}-\varphi_{0}\right)}, \quad T^{\prime}=\frac{\left(T-T_{1}\right)}{\left(T_{0}-T_{1}\right)} .
\end{array}
$$

There after dropping the dashes ( ' ) for simplicity

Equations (2.1) - (2.4) in non dimensional form can be written as

$$
\begin{aligned}
& \nabla \cdot \boldsymbol{q}=0, \\
& 0=-\nabla p-\boldsymbol{q}_{a}-\operatorname{Rm}(1+\delta h(z)) \hat{e}_{z}+\operatorname{Ra}(1+\delta h(z)) T \hat{e}_{z}-\operatorname{Rn}(1+\delta h(z)) \varphi \hat{e}_{z}, \\
& \frac{\partial T}{\partial t}+\boldsymbol{q} \cdot \nabla T=\nabla^{2}\left(\eta \nabla_{h}^{2}+\frac{\partial^{2}}{\partial z^{2}}\right) T+\frac{N_{B}}{\mathrm{Le}} \nabla \varphi \cdot \nabla T+\frac{N_{A} N_{B}}{\mathrm{Le}} \nabla T \cdot \nabla T,
\end{aligned}
$$




$$
\frac{1}{\sigma} \frac{\partial \varphi}{\partial t}+\frac{1}{\varepsilon} \boldsymbol{q} \cdot \nabla \varphi=\frac{1}{\mathrm{Le}} \nabla^{2} \varphi+\frac{N_{A}}{\mathrm{Le}} \nabla^{2} T
$$

where

$\mathrm{Le}=\frac{\kappa_{\mathrm{T}}}{D_{B}}$, Lewis number; $\boldsymbol{q}_{a}=\left(\frac{1}{\xi} u, \frac{1}{\xi} v, w\right)$, anisotropic modified velocity vector; $\xi=\frac{K_{x}}{K_{\mathrm{z}}}$, mechanical anisotropy parameter; $\eta=\frac{\kappa_{T x}}{\kappa_{T z}}$, thermal anisotropy parameter; $\operatorname{Ra}=\frac{\rho g \alpha d\left(T_{0}-T_{1}\right)}{\mu \kappa}$, Rayleigh Darcy Number; $\mathrm{Rm}=\frac{\rho_{p} \varphi_{0}+\rho\left(1-\varphi_{0}\right) g d}{\mu \kappa}$, density Rayleigh number; $\mathrm{Rn}=\frac{\left(\rho_{p}-\rho\right)\left(\varphi_{1}-\varphi_{0}\right) g d}{\mu \kappa}$, concentration Rayleigh number; $N_{A}=\frac{D_{T}\left(T_{0}-T_{1}\right)}{D_{B} T_{1}\left(\varphi_{1}-\varphi_{0}\right)}$, modified diffusivity ratio; $N_{B}=\frac{(\rho c)_{p}\left(\varphi_{1}-\varphi_{0}\right)}{(\rho c)_{f}}$, modified particle- density ratio.

The dimensionless boundary conditions are given as

$$
\begin{aligned}
& w=0, \quad T=T_{0}, \quad \varphi=\varphi_{0} \quad \text { at } \quad z=0, \quad \text { and } \\
& w=0, \quad T=T_{1}, \quad \varphi=\varphi_{1} \quad \text { at } \quad z=1 .
\end{aligned}
$$

The basic state was assumed to be quiescent and is given by

$$
u=v=w=0, \quad p=p(z), \quad T=T_{b}(z), \quad \varphi=\varphi_{b}(z) .
$$

Approximate solution as given by Kuznetsov and Nield (2010a; b), Nield and Kuznetsov (2009, $2011 \mathrm{a} ; \mathrm{b})$ is

$$
T_{b}=1-z, \quad \text { and } \quad \varphi_{b}=z
$$

To study the stability of the system, we superimposed infinitesimal perturbations on the basic state, which are of the forms

$$
\begin{array}{lll}
q(u, v, w)=0+q^{\prime}(u, v, w), & T=T_{b,}+T^{\prime}, & \varphi=\varphi_{b}+\varphi^{\prime}, \\
p=p_{b}+p^{\prime}, \quad \text { with } & T_{b}=1-z, & \varphi_{b}=z .
\end{array}
$$

Thus the linearized perturbation (neglecting the product of the prime quantities) equations are [dashes ( ' ) are suppressed for convenience]

$$
\begin{aligned}
& \nabla \cdot \boldsymbol{q}=0, \\
& 0=-\nabla p-q_{a}+\operatorname{Ra}(1+\delta h(z)) T \hat{e}_{z}-\operatorname{Rn}(1+\delta h(z)) \varphi \hat{e}_{z},
\end{aligned}
$$




$$
\begin{aligned}
& \frac{\partial T}{\partial t}-w=\left(\eta \nabla_{h}^{2}+\frac{\partial^{2}}{\partial z^{2}}\right) T+\frac{N_{B}}{\mathrm{Le}}\left(\frac{\partial T}{\partial z}-\frac{\partial \varphi}{\partial z}\right)-\frac{2 N_{A} N_{B}}{\mathrm{Le}} \frac{\partial T}{\partial z} \\
& \frac{1}{\sigma} \frac{\partial \varphi}{\partial t}+\frac{1}{\varepsilon} w=\frac{1}{\mathrm{Le}} \nabla^{2} \varphi+\frac{N_{A}}{\mathrm{Le}} \nabla^{2} T .
\end{aligned}
$$

It will be noted that the parameter $R_{m}$ is not involved in these and subsequent equations. It is just a measure of the basic static pressure gradient.

Eliminating $P$ from Eq.(2.14) by operating with $e_{z} \cdot \operatorname{curl}$ curl and using the identity curl curl $=\operatorname{grad}$ $\operatorname{div}-\nabla^{2}$, we get

$$
\left(\nabla_{H}^{2}+\frac{1}{\xi} \frac{\partial^{2}}{\partial z^{2}}\right) w=\operatorname{Ra}(1+\delta h(z)) \nabla_{H}^{2} T-R n(1+\delta h(z)) \nabla_{H}^{2} \varphi
$$

where $\nabla_{H}^{2}$, is the two-dimensional Laplacian operator on the horizontal plane.

\section{Normal modes and stability analysis} the form

Analyzing the disturbances into the normal modes and assuming that the perturbed quantities are of

$$
[w, T, \varphi]=[W(z), \Theta(z), \Phi(Z)] \exp \left(i k_{x} x+i k_{y} y+n t\right)
$$

where $k_{x,}, k_{y}$ are wave numbers in the $x$ and $y$ direction and $n$ is the growth rate of disturbances.

Using Eq.(3.1), Eqs (2.15) - (2.17) become

$$
\begin{aligned}
& \left(\frac{1}{\xi} D^{2}-a^{2}\right) W+a^{2} \operatorname{Ra}(1+\delta h(z)) \Theta-a^{2} \operatorname{Rn}(1+\delta h(z)) \Phi=0, \\
& W+\left(D^{2}-\eta a^{2}-n+\frac{N_{A}}{\mathrm{Le}} D-\frac{2 N_{A} N_{B}}{\mathrm{Le}} D\right) \Theta-\frac{N_{B}}{\mathrm{Le}} D \Phi=0, \\
& \frac{W}{\varepsilon}-\frac{N_{A}}{\mathrm{Le}}\left(D^{2}-a^{2}\right) \Theta-\left(\frac{1}{\mathrm{Le}}\left(D^{2}-a^{2}\right)-\frac{n}{\sigma}\right) \Phi=0
\end{aligned}
$$

where $D=\frac{d}{d z}$ and $a^{2}=k_{x}^{2}+k_{y}^{2}$ is the resultant dimensionless wave number. conditions

Equations (3.2) to (3.4) are subject to stress-free, isothermal, isonano concentration boundary

$$
\begin{array}{ll}
W=0, \quad D^{2} W=0, \quad \Theta=0, \quad \Phi=0 \quad \text { at } \quad z=0 \quad \text { and } \\
W=0, \quad D^{2} W=0, \quad \Theta=0, \quad \Phi=0 \quad \text { at } \quad z=1 .
\end{array}
$$


We assume the solution to $W, \Theta$ and $\Phi$ is of the form

$$
W=W_{0} \quad \sin \pi z \quad \Theta=\Theta_{0} \sin \pi z, \quad \Phi=\Phi_{0} \sin \pi z,
$$

which satisfies boundary conditions (3.5).

Substituting solution (3.6) into Eqs (3.2) - (3.4), integrating each equation from $z=0$ to $z=1$, and performing some integrations by parts, we obtain the following matrix equation

$$
\left[\begin{array}{llc}
\left(\frac{\pi^{2}}{\xi}+a^{2}\right) & -a^{2}(1+\delta h(z)) \mathrm{Ra} & a^{2}(1+\delta h(z)) \mathrm{Rn} \\
1 & -\left(\pi^{2}+\eta a^{2}+n\right) & 0 \\
\frac{1}{\varepsilon} & \frac{N_{A}}{\mathrm{Le}}\left(\pi^{2}+a^{2}\right) & \frac{\left(\pi^{2}+a^{2}\right)}{\mathrm{Le}}+\frac{n}{\sigma}
\end{array}\right]\left[\begin{array}{l}
W_{0} \\
\Theta_{0} \\
\Phi_{0}
\end{array}\right]=\left[\begin{array}{l}
0 \\
0 \\
0
\end{array}\right] .
$$

The non-trivial solution of the above matrix requires that

$$
\mathrm{Ra}=\frac{1}{a^{2}}\left(\frac{\pi^{2}}{\xi}+a^{2}\right)\left(\pi^{2}+\eta a^{2}+n\right)(1+\delta h(z))^{-1}-\frac{\frac{N_{A}}{\mathrm{Le}}\left(\pi^{2}+a^{2}\right)+\frac{\pi^{2}+\eta a^{2}+n}{\varepsilon}}{\left(\frac{\pi^{2}+a^{2}}{\mathrm{Le}}+\frac{n}{\sigma}\right)} \mathrm{Rn} .
$$

For neutral stability the real parts of $n$ are zero. Hence we now put $n=i \omega$, (where $\omega$ is a real and dimensionless quantity) in Eq.(3.7) and we get

$$
\mathrm{Ra}=\frac{1}{a^{2}}\left(\frac{\pi^{2}}{\xi}+a^{2}\right)\left(\pi^{2}+\eta a^{2}+i \omega\right)(1+\delta h(z))^{-1}-\frac{\frac{N_{A}}{\mathrm{Le}}\left(\pi^{2}+a^{2}\right)+\frac{\pi^{2}+\eta a^{2}+i \omega}{\varepsilon}}{\left(\frac{\pi^{2}+a^{2}}{\mathrm{Le}}+\frac{i \omega}{\sigma}\right)} \mathrm{Rn} .
$$

\section{Stationary convection}

For stationary convection $\omega=0$, Eq.(3.8) reduces to

$$
\mathrm{Ra}=\frac{1}{a^{2}}\left(\frac{\pi^{2}}{\xi}+a^{2}\right)\left(\pi^{2}+\eta a^{2}\right)(1+\delta h(z))^{-1}-\frac{\frac{N_{A}}{\mathrm{Le}}\left(\pi^{2}+a^{2}\right)+\frac{\pi^{2}+\eta a^{2}}{\varepsilon}}{\frac{\pi^{2}+a^{2}}{\mathrm{Le}}} \mathrm{Rn} .
$$

For the isotropic porous medium and constant gravity, i.e., if $\xi=\eta=1, \delta h(z)=0$, then Eq.(4.1) gives 


$$
\mathrm{Ra}=\frac{1}{a^{2}}\left(\pi^{2}+a^{2}\right)^{2}-\left(N_{A}+\frac{\mathrm{Le}}{\varepsilon}\right) \mathrm{Rn} .
$$

This is the same result as derived by Nield and Kuznetsov (2009; 2010b).

The minimum of Eq.(4.2) is attained at $a=\pi$, so that the critical Rayleigh number $(\mathrm{Ra})_{c}$ is given by

$$
(\mathrm{Ra})_{c}=4 \pi^{2}-\mathrm{Rn}\left(N_{A}+\frac{\mathrm{Le}}{\varepsilon}\right)
$$

It is the same result as obtained by Nield and Kuznetsov (2009), Kuznetsov and Nield (2010b) for the particular case.

In the absence of nanoparticles we obtain the critical Rayleigh -Darcy number given by $(\mathrm{Ra})_{c}=4 \pi^{2}$. This is the classical result obtained by Lapwood (1948) for regular fluids.

\section{Oscillatory convection}

For oscillatory convection we have $\omega \# 0$, thus on equating the real and imaginary parts of Eq.(3.8), we have

$$
\begin{aligned}
& a^{2} \operatorname{Ra}\left(\frac{\pi^{2}+a^{2}}{\mathrm{Le}}\right)+a^{2} \operatorname{Rn}\left(\frac{N_{A}}{\mathrm{Le}}\left(\pi^{2}+a^{2}\right)+\frac{\left(\pi^{2}+a^{2}\right)}{\varepsilon}\right)= \\
& =\left(\frac{\pi^{2}}{\xi}+a^{2}\right)\left(\left(\pi^{2}+\eta a^{2}\right)\left(\frac{\pi^{2}+a^{2}}{\mathrm{Le}}\right)-\frac{\omega^{2}}{\sigma}\right)(1+\delta h(z))^{-1},
\end{aligned}
$$

and

$$
\omega\left(\frac{a^{2} \mathrm{Ra}}{\sigma}+\frac{a^{2} \mathrm{Rn}}{\varepsilon}-(1+\delta h(z))^{-1}\left(\frac{\pi^{2}}{\xi}+a^{2}\right)\left(\frac{\pi^{2}+\eta a^{2}}{\sigma}+\frac{\pi^{2}+a^{2}}{\mathrm{Le}}\right)\right)=0 .
$$

From Eq.(5.1), the frequency of the oscillatory mode is given as

$$
\begin{aligned}
& \left(\frac{\pi^{2}}{\xi}+a^{2}\right) \frac{\omega^{2} \mathrm{Le}}{\sigma}=(1+\delta h(z))^{-1}\left(\left(\pi^{2}+\eta a^{2}\right) \pi^{2}+a^{2}\right)\left(\frac{\pi^{2}}{\xi}+a^{2}\right)+ \\
& -\left(a^{2} \operatorname{Ra}\left(\pi^{2}+a^{2}\right)+a^{2} \operatorname{Rn}\left(N_{A}\left(\pi^{2}+a^{2}\right)+\frac{\mathrm{Le}\left(\pi^{2}+a^{2}\right)}{\varepsilon}\right) .\right.
\end{aligned}
$$

In order for $\omega$ to be real it is necessary that

$$
\mathrm{Ra}+\mathrm{Rn}\left(N_{A}+\frac{\mathrm{Le}}{\varepsilon}\right) \leq \frac{\left(\pi^{2}+\eta a^{2}\right)}{a^{2}}\left(\frac{\pi^{2}}{\xi}+a^{2}\right)(1+\delta h(z))^{-1} .
$$


Thus in these cases the critical value of Ra is independent of both $N_{A}$ and $N_{B}$, but the frequency does depend on $N_{A}$.

As the wave number ' $a$ ' varies, the minimum of Eqs (5.2) and (5.4) is attained at $a=\pi$, so that the critical Rayleigh number is attained when

$$
\frac{\mathrm{Ra}}{\sigma}+\frac{\mathrm{Rn}}{\varepsilon}=\pi^{2}\left(\frac{1+\xi}{\xi}\right)\left(\frac{1+\eta}{\sigma}+\frac{2}{\mathrm{Le}}\right)(1+\delta h(z))^{-1}
$$

In order for $\omega$ to be real it is necessary that

$$
\mathrm{Ra}+\mathrm{Rn}\left(N_{A}+\frac{\mathrm{Le}}{\varepsilon}\right) \leq \pi^{2}\left(\frac{1+\xi}{\xi}\right)(1+\eta)(1+\delta h(z))^{-1} .
$$

For the isotropic porous medium and constant gravity, i.e., if $\xi=\eta=1, \delta h(z)=0$, Eqs (5.5) and (5.6) reduce to

$$
\frac{\mathrm{Ra}}{\sigma}+\frac{\mathrm{Rn}}{\varepsilon}=4 \pi^{2}\left(\frac{1}{\sigma}+\frac{1}{\mathrm{Le}}\right)
$$

In order for $\omega$ to be real it is necessary that

$$
\operatorname{Ra}+\operatorname{Rn}\left(N_{A}+\frac{\mathrm{Le}}{\varepsilon}\right) \leq 4 \pi^{2}
$$

These were the same results as obtained by Nield and Kuznetsov (2011b) for the particular case.

\section{Results and discussion}

We present stability curves for the stationary Rayleigh number Ra versus $z$ for the mechanical anisotropy parameter $\xi$, thermal anisotropy parameter $\eta$ and variable gravity parameter for the fixed values of other parameters.

Figure 1 indicates the effect of the mechanical anisotropy parameter $\xi$, and variable gravity parameter on the stationary convection and it is found that the Rayleigh number decreases with an increase in the value of the mechanical anisotropy parameter $\xi$, thus the mechanical anisotropy parameter $\xi$ is to advance the onset of stationary convection.

Figure 2 indicates the effect of the thermal anisotropy parameter $\eta$, on stationary convection and it is found that the critical Rayleigh number increases with an increase in the value of the thermal anisotropy parameter $\eta$, indicating that the effect of the thermal anisotropy parameter $\eta$ is to inhibit the onset of convection. Also, it is found that the value of the stationary Rayleigh number increases when we take a decreasing gravity profile, i.e., when the variable gravity parameter is $h(z)=z^{2}-2 z$ (convex one), $h(z)=-$ $z, h(z)=-z^{2}$ (concave one), while the value of the stationary Rayleigh number decreases when we take an increasing gravity profile, i.e., when the variable gravity parameter is $h(z)=z$. Thus the decreasing gravity parameter has a stabilizing effect while the increasing gravity parameter has a destabilizing effect on stationary convection. 


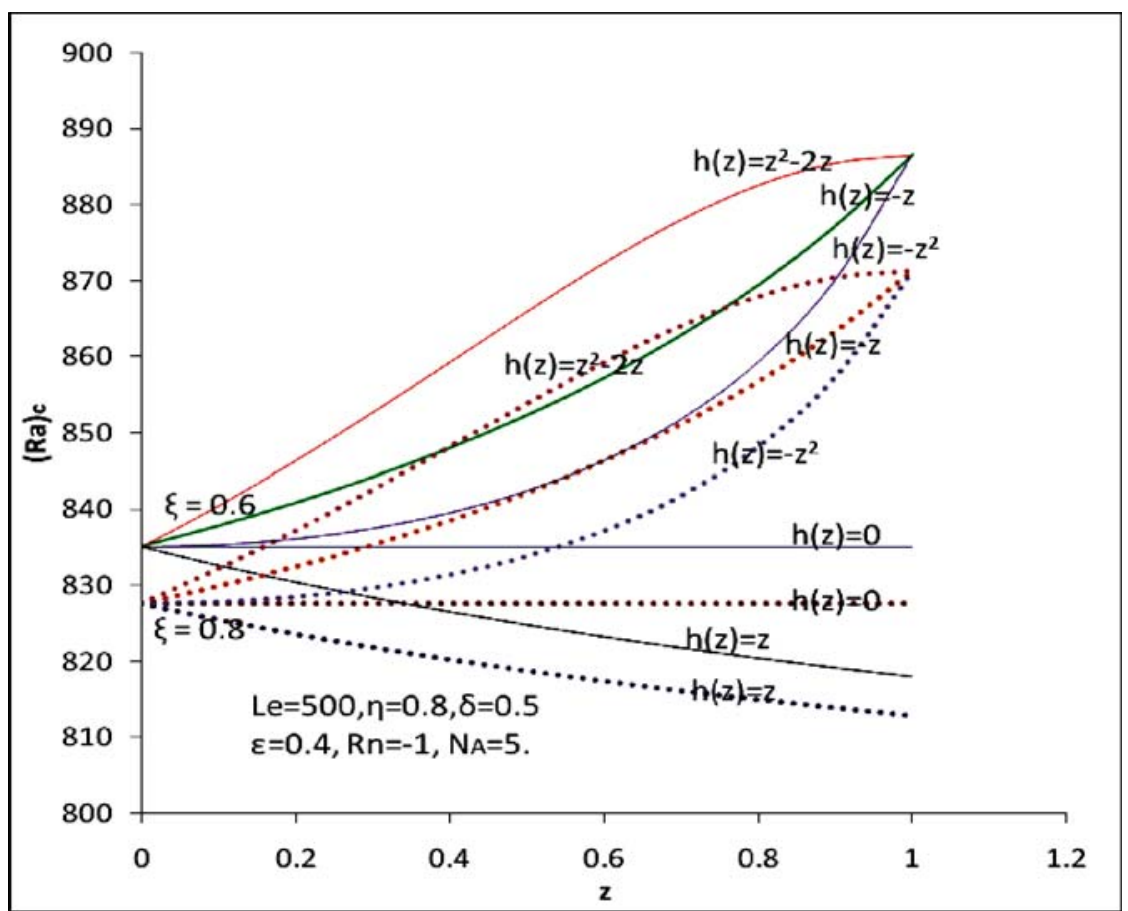

Fig.1. Mechanical anisotropy and variable gravity effects on the critical Rayleigh number.

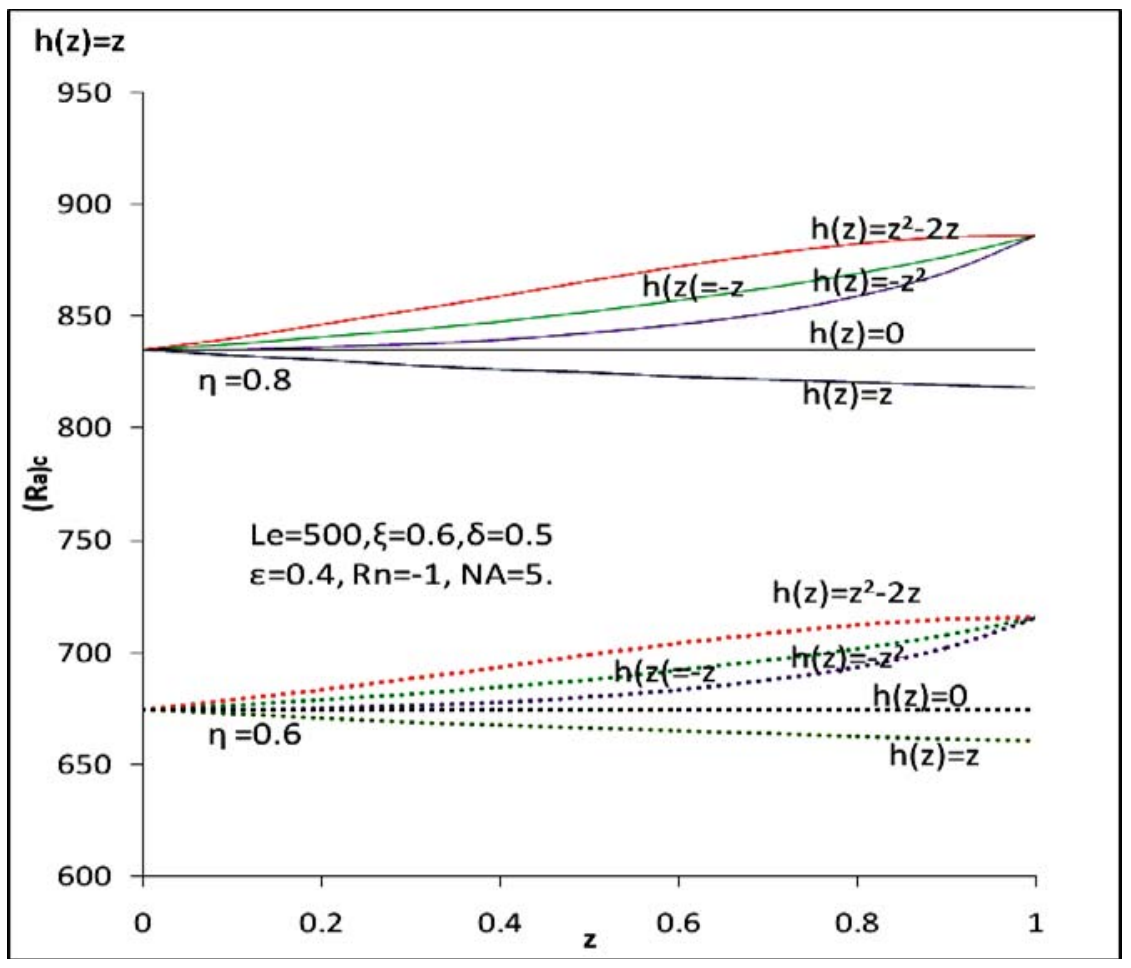

Fig.2. Thermal anisotropy and variable gravity effects on the critical Rayleigh number. 


\section{Conclusions}

We considered variable gravity effects on thermal instability in a horizontal layer of a nanofluid in an anisotropic porous medium. We draw the following conclusions:

(i) The critical cell size is not a function of any thermo physical properties of the nanofluid.

(ii) The critical value of Ra is independent of both $N_{A}$ and $N_{B}$, but the frequency does depend on $N_{A}$.

(iii) The mechanical anisotropy parameter $\xi$ is to advance the onset of oscillatory non-oscillatory convection.

(iv) The thermal anisotropy parameter $\eta$ is to inhibit the onset of oscillatory and non-oscillatory convection.

(v) The decreasing gravity parameter has a stabilizing effect while the increasing gravity parameter has a destabilizing effect on stationary convection.

\section{Acknowledgment}

The first author thanks the University Grants Commission, New Delhi, India, for financial support under Minor Research Project [F. No. 8-3(123)/2011(MRP/NRCB].

\section{Nomenclature}

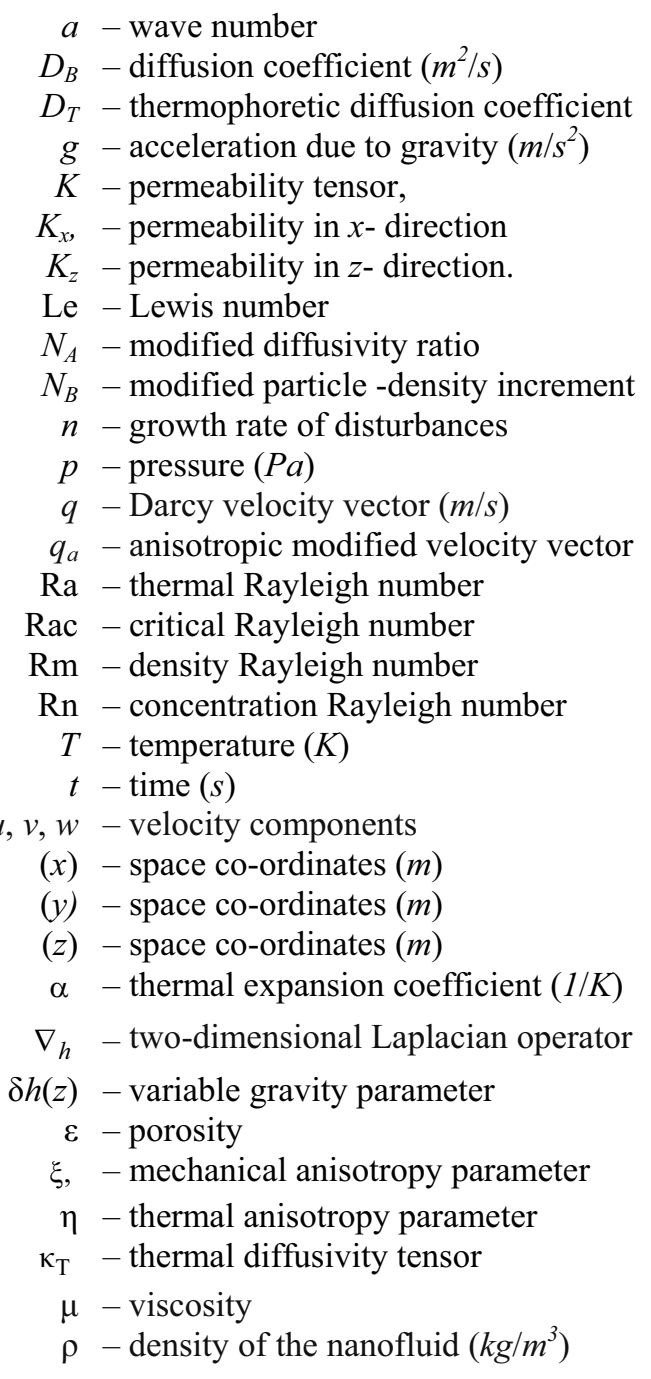


$\rho_{\mathrm{f}}-$ density of base fluid

$\rho_{\mathrm{p}}-$ density of nano particles

$\varphi-$ volume fraction of the nanoparticles

$\psi-$ stream function

$\omega-$ dimensional frequency

\section{Superscripts}

' - non dimensional variables

\section{Subscripts}

$$
\begin{aligned}
& 0 \text { - lower boundary } \\
& 1 \text { - upper boundary } \\
& f \text { - fluid } \\
& h \text { - horizontal plane } \\
& p \text { - particle }
\end{aligned}
$$

\section{References}

Alex S.M., Prabhamani R.P. and Vankatakrishan K.S. (2001): Variable gravity effects on thermal instability in a porous medium with internal heat source and inclined temperature gradient. - Fluid Dynamics Research, vol.29, pp.1-6.

Alloui Z., Vasseur P. and Reggio M. (2010): Natural convection of nanofluids in a shallow cavity heated from below. - International Journal of Thermal Science, online xxx, pp.1-9.

Buongiorno J. (2006): Convective transport in nanofluids. - ASME Journal of Heat Transfer, vol.128, pp.240-250.

Chand R. (2011): Effect of suspended particles on thermal instability of Maxwell visco-elastic fluid with variable gravity in porous medium. - Antarctica J. Math, vol.8(6), pp.487-497.

Chand R. (2012): Thermal instability of rotating Maxwell visco-elastic fluid with variable gravity in porous medium. Journal of the Indian Math. Soc. Accepted for publications.

Chand R. (2012): Thermal instability of rotating nanofluid. - Int. J. of Appl. Math and Mech. Accepted for publication.

Chand R. and Rana G.C. (2012): On the onset of thermal convection in rotating nanofluid layer saturating a DarcyBrinkman porous medium. - Int. J. Heat Mass Transf., Accepted for publication.

Chandrasekhar S. (1981): Hydrodynamic and Hydromagnetic Stability. - New York: Dover Publication.

Choi S. (1995): Enhancing thermal conductivity of fluids with nanoparticles. - In: Siginer D.A., Wang, H.P. (eds.) Developments and Applications of Non-Newtonian Flows, ASME FED-vol.231/MD-vol.66, pp.99-105.

Dhananjay Agrawal G.S. and Bhargava R (2010): Rayleigh Bénard convection in nanofluid. - Int. J. of Appl. Math and Mech. vol.7(2), pp.61-76.

Ingham D.D. and Pop L. (1981): Transport Phenomena in Porous Media. - New York: Elsvier.

Kim S.J., Bang I.C., Buongiorno J and Hu L.W. (2007): Study of pool boiling and critical heat flux enhancement in nanofluids. - Bulletin of the Polish Academy of Sciences-Technical Sciences, vol.55,2, pp.211-216.

Kuznetsov A.V. and Nield D.A. (2010a): Effect of local Thermal non-equilibrium on the onset of convection in a porous medium layer saturated by a nanofluid. - Transport in Porous Media, vol.83, pp.425-436.

Kuznetsov A.V. and Nield D.A. (2010b): Thermal instability in a porous medium layer saturated by a nanofluid: Brinkman model. - Transp. Porous Medium, vol.81, pp.409-422.

Kuznetsov A.V. and Nield D.A. (2010c): The onset of double-diffusive nanofluid convection in a layer of a saturated porous medium. - Transport in Porous Media, vol.85,3, pp.941-951.

Lapwood E.R. (1948): Convection of a fluid in porous medium. - Proc. Camb. Phil. Soc., vol.44, pp.508-519. 
Malashetty M.S. and Kollur P. (2011): The onset of double diffusive convection in a couple stress fluid saturated anisotropic porous layer. - Transp. Porous Med., vol.86, pp.435-459.

Nield D.A. and Bejan A. (2006): Convection in porous medium. - New York: Springer.

Nield D.A. and Kuznetsov A.V. (2010): The onset of convection in a horizontal nanofluid layer of finite depth. European Journal of Mechanics - B/Fluids, vol.29, pp.217-223.

Nield D.A. and Kuznetsov A.V. (2011a): The onset of double-diffusive convection in a nanofluid layer. - International Journal of Heat and Fluid Flow, vol.32, pp.771-776.

Nield D.A. and Kuznetsov A.V. (2011b): The effect of vertical through flow on thermal instability in a porous medium layer saturated by a nanofluid. - Transport in Porous Media vol.87, pp.765-775.

Nield D.A. and Kuznetsov, A.V. (2009): Thermal instability in a porous medium layer saturated by a nanofluid. - Int. J. Heat Mass Transf. vol.52, pp 5796-5801.

Pradhan G.K., Samal P.C. and Tripathy U.K. (1989): Thermal stability of a fluid layer in a variable gravitational field. - Indian J. Pure Appl. Math., vol.20(7), pp.736-745.

Tzou D.Y. (2008a): Instability of nanofluids in natural convection. - ASME J. Heat Transf., vol.130, pp.072401.

Tzou D.Y. (2008b): Thermal instability of nanofluids in natural convection. - Int. J. Heat Mass Transf., vol.51, pp.2967-2979.

Vadasz P. (2006): Heat conduction in nanofluid suspensions. - ASME J. Heat Transf., vol.128, pp.465-477.

Vafai K.A. and Hadim H.A. (2000): Hand Book of Porous Media. - New York: M. Decker.

Wooding R.A. (1960): Rayleigh instability of a thermal boundary layer in flow through a porous medium. - J. Fluid Mech., vol.9, pp.183-192.

Received: June 5, 2012

Revised: May 28, 2013 